artery disease. The first, multiple aortic crossclamping, was a tried and true method in our hospital until recently. It involved the use of an aortic clamp that we have shown generates more pressure on the walls of the aorta, as well as multiple applications of partial occluding clamps to the aorta. Our newer technique involves a softer aortic crossclamp and no partial occluding clamps at all. The dramatic difference in the 6-month neurocognitive result amply supports the newer technique.

The authors of the letter were disappointed in the lost opportunity of grading athroma on transesophageal echocardiogram and comparing the results with neurologic outcomes. We reported in our Methods section that each patient received an epiaortic ultrasound scan of the ascending aorta and a transesophageal echocardiogram of the descending aorta. We regret that we failed to publish the fact that there were no statistically significant correlations between ascending or descending aortic atheroma and neurocognitive outcomes in any of the groups. We stated in our Methods section that we believed it was unethical to randomize patients with grade 5 atheromata to multiple aortic clamping, and we still believe that this was the correct decision, as it did not significantly influence outcome.

My coauthors and I would like to thank Drs Purohit and Zacharias for their thoughtful comments. We appreciate their careful reading of our manuscript.

John W. Hammon, Jr, MD

Wake Forest University School of Medicine Department of Cardiothoracic Surgery Medical Center Blvd Winston-Salem, NC 27157-1096 doi:10.1016/j.jtcvs.2006.05.022

\section{Primary sutureless repair of total anomalous pulmonary venous connection: The value of intrapleural hilar reapproximation}

To the Editor:

I read with great interest the recent article of Meadows and colleagues ${ }^{1}$ describing an elegant approach to the repair of an obstructed total anomalous pulmonary venous connection in a critically ill neonate. As clearly demonstrated, the venous confluence was very poorly developed in the patient, necessitating the use of primary sutureless technique. ${ }^{1}$ This technique of suturing the atrium directly to the posterior pericardium around the opened confluence of the pulmonary veins ${ }^{2,3}$ is now frequently used for primary repair of the total anomalous pulmonary venous connection in the adhesionfree pericardial cavity. ${ }^{4}$ Despite the absence of retrocardiac adhesions, operative mortality is not increased with sutureless technique as a primary procedure relative to sutureless repair as a reoperation. ${ }^{4}$

When used for primary repair in adhesionfree thin pericardium in a neonate, how- ever, the sutureless technique should be applied with caution. A thorough understanding of the anatomic relationship of the pulmonary venous confluence, posterior pleuropericardial junction, and phrenic nerve is required to perform a complication-free repair. ${ }^{5}$ It is crucial to open the venous confluence widely to ensure an unobstructed connection. Longitudinal incision in the pulmonary veins may, however, occasionally violate the thin pleuropericardial junction (Figure 1, A), with subsequent bleeding into the pleural space. ${ }^{4}$ This com-

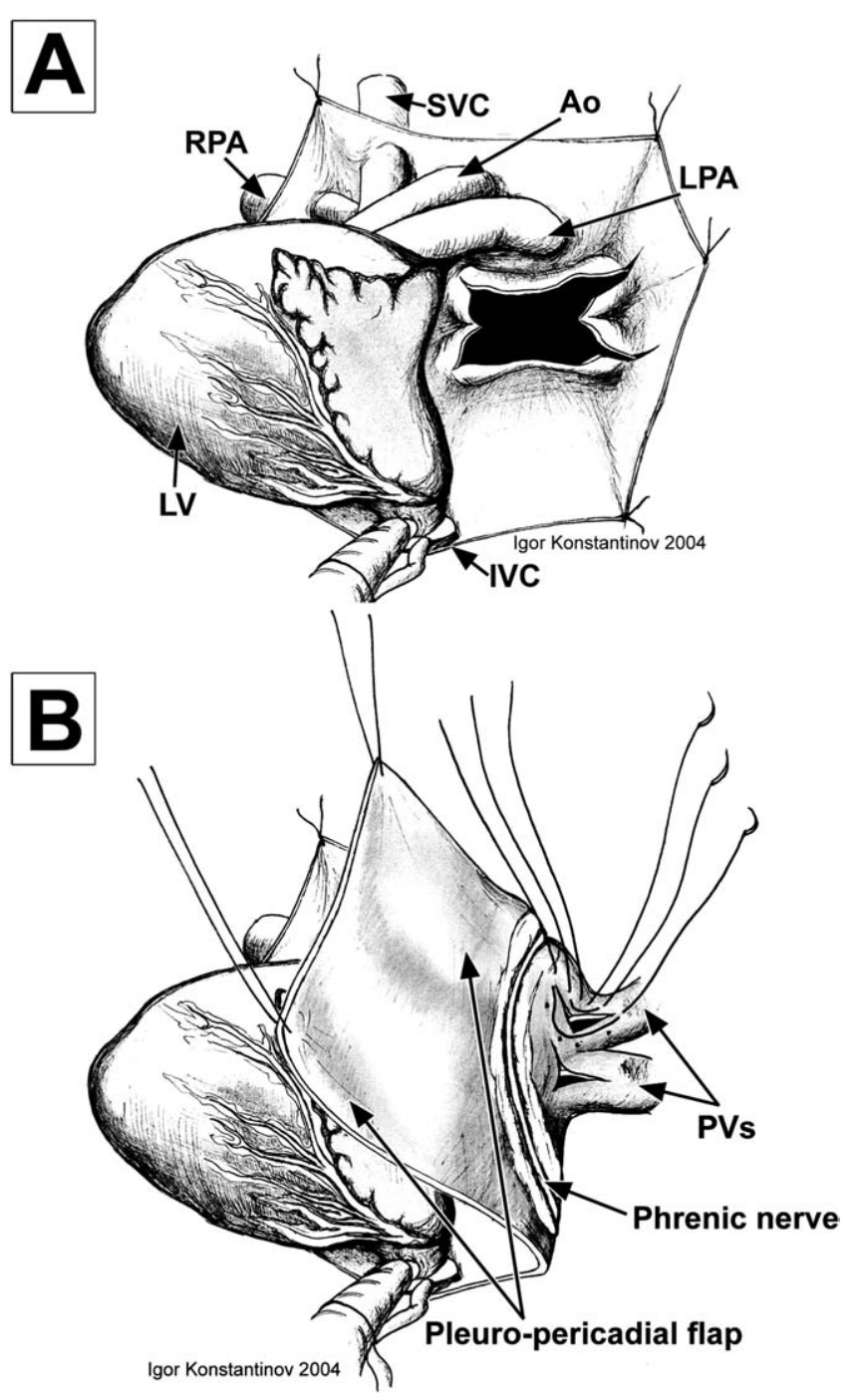

Figure 1. Transpericardial incision in pulmonary veins $(A)$ may result in violation of thin pleuropericardial junction (B). Pleuropericardial flap is then retracted toward midline, exposing anterior aspect of pulmonary hilum (B). Parietal and visceral pleura can then be approximated to achieve hemostasis. RPA, Right pulmonary artery; SVC, superior vena cava; $A$ o, aorta; $L P A$, left pulmonary artery, $L V$, left ventricle; $I V C$, inferior vena cava; $P V s$, pulmonary veins. 
plication can be successfully managed by intrapleural hilar reapproximation technique (Figure 1, B). The pleuropericardial flap is retracted toward the midline, and the pleura is reapproximated to achieve hemostasis.

This technique must be in the armamentarium of every pediatric cardiac surgeon who intends to apply the sutureless technique as a primary repair in adhesion-free pericardium.

Igor E. Konstantinov, $M D, P h D$ Division of Cardiovascular Surgery Hospital for Sick Children Toronto, Ontario, Canada

\section{References}

1. Meadows J, Marshall AC, Lock JE, Scheurer M, Laussen PC, Bacha EA. A hybrid approach to stabilization and repair of obstructed total anomalous pulmonary venous connection in a critically ill newborn infant. J Thorac Cardiovasc Surg. 2006;131:e1-2.

2. Lacour-Gayet F, Rey C, Planche C. Pulmonary vein stenosis. Description of a sutureless surgical procedure using the pericardium in situ. Arch Mal Coeur Vaiss. 1996;89:633-6.

3. Caldarone CA, Najm HK, Kadletz M, Smallhorn JF, Freedom RM, Williams WG, et al. Relentless pulmonary vein stenosis after repair of total anomalous venous drainage. Ann Thorac Surg. 1998;66:1514-20.

4. Yun TJ, Coles JG, Konstantinov IE, Al-Radi OO, Wald RM, Guerra V, et al. Conventional and sutureless techniques for management of the pulmonary veins, evolution of indications from postrepair pulmonary vein stenosis to primary pulmonary vein anomalies. $J$ Thorac Cardiovasc Surg. 2005;129:167-74.

5. Freedom RM, Yoo SJ, Coles JG, Konstantinov IE. Total anomalous pulmonary venous connections. In: Freedom RM, editor. The natural and modified history of congenital heart disease. New York: Future Publishing; 2003. p. 282-9.

$$
\text { doi:10.1016/j.jtcvs.2006.05.038 }
$$

\section{Reply to the Editor:}

I thank Dr Kostantinov for his thoughtful letter. I entirely agree with the first half of his statement. For complex total anomalous pulmonary venous connection, especially with a poorly developed confluence, the primary sutureless technique (in which the opened edge of the posterior left atrium is sutured to the posterior pericardium, leaving the filleted open pulmonary venous confluence to drain freely in the neo-left atrium) offers many advantages. It has become my default technique when the traditional pulmonary venous confluence to left atrium suture anastomosis is impractical. As of June 2006 (11 months after the operation), the subject of our report is growing well, with no evidence of pulmonary venous obstruction according to echocardiography. Clearly, I also agree that "a thorough understanding of the anatomic relationship of the pulmonary venous confluence, posterior pleuropericardial junction, and phrenic nerve is required to perform a complication-free repair," and that it "It is crucial to open the venous confluence widely to ensure an unobstructed connection." Those are surgical tenets. To incise the pleuropericardial junction inadvertently, however, one must be cutting quite deeply into the pulmonary veins themselves. That is something that is rarely required. Usually, an incision of a few millimeters into each vein will suffice. Occasionally, the pulmonary veins themselves are diffusely stenotic and require a deeper incision. That is exceptional, however, and usually occurs in a reoperative situation. I would also caution against using sutures at the level of the pleuropericardial junction and pulmonary veins (Konstantinov's Figure 1, $B$ ). The risk of recurrent or residual pulmonary venous stenosis is ever present in these patients, and even a 1 - to $2-\mathrm{mm}$ encroachment into the pulmonary venous lumen by a suture intended for hemostasis may have catastrophic consequences. If one feels that a suture at this level is absolutely necessary, then the lumen of the veins should be inspected after tying it.

Emile Bacha, MD Harvard Medical School Boston, Mass doi:10.1016/j.jtcvs.2006.05.037

\section{Subclavian artery from ascending aorta or as the first branch of the aortic arch: Another variant of persistent fifth aortic arch \\ To the Editor:}

We read with interest the articles by Krishnamoorthy and colleagues ${ }^{1}$ and by Krishnan and colleagues ${ }^{2}$ reporting 3 cases of tetralogy of Fallot with right aortic arch and left subclavian artery originating from the ascending aorta. Both groups claim these to be the only such cases reported in the literature. Although we agree that this anatomic set is rather uncommon, we consider it to be a well-established malformation, with several similar cases described previously. ${ }^{3}$
The presumed uniqueness of such cases has arisen from the incomplete understanding of aortic arch development in the context of these malformations and consequent misinterpretation. Krishnamoorthy and colleagues ${ }^{1}$ propose persistence of the left fourth arch in the setting of partial double arch as the basis of the anomaly. In the normal arrangement, however, both fourth aortic arches are always persistent, one forming the first segment of the subclavian artery at its origin from the innominate artery and the other giving rise to the distal aortic arch between the carotid and subclavian artery. When the subclavian artery arises from the ascending aorta or as the first branch of the aortic arch, we believe exactly the opposite occurs; in the presence of a right aortic arch, the fourth left arch is interrupted, and the subclavian artery is continuous with the ascending aorta through a persistent left fifth aortic arch and possibly a short segment of the dorsal aorta, as originally proposed by Moes and colleagues. ${ }^{3,4}$

Conversely, Krishnan and colleagues ${ }^{2}$ advocate incomplete cephalad migration of the seventh intersegmental artery, describing it as an aberrant subclavian artery arising from the ascending aorta. The subclavian artery is not aberrant, however, and the left dorsal aorta, which connects the subclavian artery to the descending aorta in the presence of a truly aberrant subclavian artery, is normally absent.

Persistence of the fifth aortic arch, first described by Van Praagh and Van Praagh, ${ }^{5}$ is a rare condition that may occur in several forms: (1) double-lumen aortic arch, with or without arch hypoplasia or coarctation; (2) with interrupted fourth homolateral arch or proximal homolateral dorsal aorta (between the attachment of the fourth and fifth arches), resulting in type A or B interrupted aortic arch, respectively; (3) with interrupted fourth heterolateral arch, resulting in connection of the subclavian artery to the ascending aorta or as the first branch of the aortic arch; and (4) systemic-topulmonary connection, with or without pulmonary or systemic obstruction. On the basis of cases described to date, we propose a concise yet comprehensive classification of the 5th persistent aortic arch, placing particular emphasis on embryological derivation and clinical relevance (Table 1). Interruption of the homolateral proximal dorsal aorta, or contralateral fourth aortic arch, results in the brachiocephalic arteries arising apparently 